\title{
ANALYSIS OF THE AXIALVECTOR DOUBLY HEAVY TETRAQUARK STATES WITH QCD SUM RULES
}

\author{
Zhi-Gang Wang 1 \\ Department of Physics, North China Electric Power University, Baoding 071003, P. R. China
}

\begin{abstract}
In this article, we construct the axialvector-diquark-scalar-antidiquark type currents to interpolate the axialvector doubly heavy tetraquark states, and study them with the QCD sum rules in details by carrying out the operator product expansion up to the vacuum condensates of dimension 10 .
\end{abstract}

PACS number: 12.39.Mk, 12.38.Lg

Key words: Tetraquark state, QCD sum rules

\section{Introduction}

The scattering amplitude for one-gluon exchange is proportional to

$$
\begin{aligned}
t_{j m}^{a} t_{k n}^{a} & =-\frac{2}{3} \frac{\delta_{j k} \delta_{m n}-\delta_{j n} \delta_{k m}}{2}+\frac{1}{3} \frac{\delta_{j k} \delta_{m n}+\delta_{j n} \delta_{k m}}{2} \\
& =-\frac{1}{3} \varepsilon_{i j m} \varepsilon_{i k n}+\frac{1}{6} S_{j m, k n}
\end{aligned}
$$

where $t^{a}=\frac{\lambda^{a}}{2}$, the $\lambda^{a}$ is the Gell-Mann matrix, $S_{j m, k n}=\delta_{j k} \delta_{m n}+\delta_{j n} \delta_{k m}$, the $i, j, k, m$ and $n$ are color indexes. The negative sign in front of the antisymmetric antitriplet $\overline{3}$ indicates the interaction is attractive while the positive sign in front of the symmetric sextet 6 indicates the interaction is repulsive, the attractive interaction favors formation of the diquarks in color antitriplet while the repulsive interaction disfavors formation of the diquarks in color sextet [1]. We can construct both the $\overline{3} \otimes 3$ type currents $\varepsilon_{i j k} \varepsilon_{i m n} Q_{j}^{T} C \Gamma Q_{k} \bar{q}_{m} \Gamma^{\prime} C \bar{q}_{n}^{\prime T}$ and the $6 \otimes \overline{6}$ type currents $S_{j k, m n} Q_{j}^{T} C \Gamma Q_{k} \bar{q}_{m} \Gamma^{\prime} C \bar{q}_{n}^{\prime T}$ satisfying Fermi-Dirac statistics to interpolating the doubly heavy tetraquark states, where the $\Gamma$ and $\Gamma^{\prime}$ are the Dirac $\gamma$ matrixes. If there really exist the $6 \otimes \overline{6}$ type doubly charmed tetraquark states, they should have much larger masses than the corresponding $\overline{3} \otimes 3$ type tetraquark states with the same quantum numbers. The color antitriplet diquarks $\varepsilon^{i j k} Q_{j}^{T} C \Gamma Q_{k}$ with $Q=c$ or $b$ only have two structures in Dirac spinor space, where $\Gamma=\gamma_{\mu}$ and $\sigma_{\mu \nu}$ for the axialvector and tensor diquarks, respectively. The axialvector diquarks $\varepsilon^{i j k} Q_{j}^{T} C \gamma_{\mu} Q_{k}$ are more stable than the tensor diquarks $\varepsilon^{i j k} Q_{j}^{T} C \sigma_{\mu \nu} Q_{k}$, it is better to choose the axialvector diquarks $\varepsilon^{i j k} Q_{j}^{T} C \gamma_{\mu} Q_{k}$ to construct the ground state doubly heavy tetraquark states.

In 2016, the LHCb collaboration observed the doubly charmed baryon state $\Xi_{c c}^{++}$in the $\Lambda_{c}^{+} K^{-} \pi^{+} \pi^{+}$mass spectrum in a $p p$ data sample collected by LHCb at $\sqrt{s}=13 \mathrm{TeV}$ with a signal yield of $313 \pm 33$, and measured the mass, but did not determine the spin [2. The $\Xi_{c c}^{++}$ maybe have the spin $\frac{1}{2}$ or $\frac{3}{2}$, we can take the diquark $\varepsilon^{i j k} c_{i}^{T} C \gamma_{\mu} c_{j}$ as basic constituent to construct the current

$$
J_{\Xi_{c c}}(x)=\varepsilon^{i j k} c_{i}^{T}(x) C \gamma_{\mu} c_{j}(x) \gamma_{5} \gamma^{\mu} u_{k}(x)
$$

or

$$
J_{\Xi_{c c}}^{\mu}(x)=\varepsilon^{i j k} c_{i}^{T}(x) C \gamma^{\mu} c_{j}(x) u_{k}(x),
$$

to study it with the QCD sum rules [3].

Up to now, no experimental candidates for the tetraquark configurations $Q Q \bar{q} \bar{q}^{\prime}$ or $q q^{\prime} \bar{Q} \bar{Q}$ have been observed. The observation of the doubly charmed baryon state $\Xi_{c c}^{++}$has led a renaissance

\footnotetext{
${ }^{1}$ E-mail: zgwang@aliyun.com.
} 
in the doubly heavy tetraquark spectroscopy. In this article, we choose the axialvector diquarks $\varepsilon^{i j k} Q_{j}^{T} C \gamma_{\mu} Q_{k}$ to construct the currents to interpolate the doubly heavy tetraquark states. There have been many works on the doubly heavy tetraquark states, such as potential quark models [4, 5, 6, 7, 8, or constituent diquark models [9, QCD sum rules [10, 11, 12, heavy quark symmetry [13, 14, 15, 16, lattice QCD [17, 18, 19], etc. If the two heavy quarks are in a long separation, the gluon exchange force between them is screened by the two light quarks, then a loosely $Q \bar{q}-Q \bar{q}^{\prime}$ type bound state is formed. On the other hand, if the two heavy quarks are in a short separation, the heavy $Q Q$ pair forms a compact point-like color source in heavy quark limit, and attracts a light $\bar{q} \bar{q}^{\prime}$ pair which serves as another compact point-like color source, then an exotic $Q Q-\bar{q} \bar{q}^{\prime}$ type tetraquark state is formed. The existence and stability of the $Q Q \bar{q} \bar{q}^{\prime}$ tetraquark states have been extensively discussed in early literatures based on the potential models [4, 5] and heavy quark symmetry [13, while the existing doubly heavy tetraquark mass spectra differ from each other in one way or the other [6, 7, 8, 9, 10, 11, 12, 14, 15, 16, 18, 19. More theoretical and experimental works are still needed.

The QCD sum rules is a powerful nonperturbative theoretical tool in studying the ground state hadrons, and has given many successful descriptions of the hadronic properties [20, 21, 22. Although the doubly heavy tetraquark states have been studied with the QCD sum rules, the energy scale dependence of the QCD sum rules has not been studied yet. In Refs. 23, 24, 25, 26, 27, we observe that in the QCD sum rules for the hidden-charm (or hidden-bottom) tetraquark states and molecular states, the integrals

$$
\int_{4 m_{Q}^{2}(\mu)}^{s_{0}} d s \rho_{Q C D}(s, \mu) \exp \left(-\frac{s}{T^{2}}\right)
$$

are sensitive to the heavy quark masses $m_{Q}(\mu)$, where the $\rho_{Q C D}(s, \mu)$ denotes the QCD spectral densities and the $T^{2}$ denotes the Borel parameters. Variations of the heavy quark masses $m_{Q}(\mu)$ or the energy scales $\mu$ lead to changes of integral ranges $4 m_{Q}^{2}(\mu)-s_{0}$ of the variable $d s$ besides the QCD spectral densities $\rho_{Q C D}(s, \mu)$, therefore changes of the Borel windows and predicted masses and pole residues. In this article, we revisit the QCD sum rules for the axialvector doubly heavy tetraquark states and choose the optimal energy scales to extract the masses.

The article is arranged as follows: we derive the QCD sum rules for the masses and pole residues of the axialvector doubly heavy tetraquark states in Sect.2; in Sect.3, we present the numerical results and discussions; and Sect.4 is reserved for our conclusion.

\section{The QCD sum rules for the axialvector doubly heavy tetraquark states}

In the following, we write down the two-point correlation functions $\Pi_{\mu \nu}^{J}(p)$ and $\Pi_{\mu \nu}^{\eta}(p)$ in the QCD sum rules,

$$
\Pi_{\mu \nu}^{J / \eta}(p)=i \int d^{4} x e^{i p \cdot x}\left\langle 0\left|T\left\{J / \eta_{\mu}(x) J / \eta_{\nu}^{\dagger}(0)\right\}\right| 0\right\rangle
$$

where

$$
\begin{aligned}
& J_{\mu}(x)=\varepsilon^{i j k} \varepsilon^{i m n} Q_{j}^{T}(x) C \gamma_{\mu} Q_{k}(x) \bar{u}_{m}(x) \gamma_{5} C \bar{s}_{n}^{T}(x), \\
& \eta_{\mu}(x)=\varepsilon^{i j k} \varepsilon^{i m n} Q_{j}^{T}(x) C \gamma_{\mu} Q_{k}(x) \bar{u}_{m}(x) \gamma_{5} C \bar{d}_{n}^{T}(x),
\end{aligned}
$$

$Q=c, b$, the $i, j, k, m, n$ are color indexes, the $C$ is the charge conjugation matrix. In the type-II diquark-antidiquark model [28, the building blocks (diquark and antidiquark) are taken as pointlike color sources, the size of the entire tetraquark is consistently larger than the size of its building blocks, the spin-spin interactions between the quarks and antiquarks in the effective Hamiltonian 
in the type-I diquark-antidiquark model [29] are neglected. The mass spectrum derived in the type-II diquark-antidiquark model is superior to that derived in the type-I diquark-antidiquark model, and is compatible with the experimental data. The tetraquark states are spatial extended objects, not point-like objects, while we choose the local currents to interpolate the tetraquark states in the QCD sum rules, and take all the quarks and antiquarks as the color sources, the finite size effects are neglected, which leads to some uncertainties.

On the phenomenological side, we insert a complete set of intermediate hadronic states with the same quantum numbers as the current operators $J_{\mu}(x)$ and $\eta_{\mu}(x)$ into the correlation functions $\Pi_{\mu \nu}^{J}(p)$ and $\Pi_{\mu \nu}^{\eta}(p)$ respectively to obtain the hadronic representation [20, 21], and isolate the ground state contributions,

$$
\begin{aligned}
\Pi_{\mu \nu}^{J / \eta}(p) & =\frac{\lambda_{Z}^{2}}{M_{Z}^{2}-p^{2}}\left(-g_{\mu \nu}+\frac{p_{\mu} p_{\nu}}{p^{2}}\right)+\cdots \\
& =\Pi_{J / \eta}\left(p^{2}\right)\left(-g_{\mu \nu}+\frac{p_{\mu} p_{\nu}}{p^{2}}\right)+\cdots,
\end{aligned}
$$

where the pole residues $\lambda_{Z}$ are defined by $\left\langle 0\left|J / \eta_{\mu}(0)\right| Z_{Q Q}(p)\right\rangle=\lambda_{Z} \varepsilon_{\mu}$, the $\varepsilon_{\mu}$ are the polarization vectors of the axialvector tetraquark states $Z_{Q Q}$.

The current $J_{\mu}(x)$ can be rewritten as

$$
\begin{aligned}
J_{\mu}(x) & =Q_{j}^{T}(x) C \gamma_{\mu} Q_{k}(x)\left[\bar{u}_{j}(x) \gamma_{5} C \bar{s}_{k}^{T}(x)-\bar{u}_{k}(x) \gamma_{5} C \bar{s}_{j}^{T}(x)\right] \\
& =\frac{1}{2}\left[Q_{j}^{T}(x) C \gamma_{\mu} Q_{k}(x)-Q_{k}^{T}(x) C \gamma_{\mu} Q_{j}(x)\right]\left[\bar{u}_{j}(x) \gamma_{5} C \bar{s}_{k}^{T}(x)-\bar{u}_{k}(x) \gamma_{5} C \bar{s}_{j}^{T}(x)\right]
\end{aligned}
$$

according to the identity $\varepsilon_{i j k} \varepsilon_{i m n}=\delta_{j m} \delta_{k n}-\delta_{j n} \delta_{k m}$ in the color space. The current $J_{\mu}(x)$ is of $\overline{3} \otimes 3$ type in both the color space and flavor space, we can also construct the current $\widetilde{J}_{\mu}(x)$ satisfying Fermi-Dirac statistics, which is of $6 \otimes \overline{6}$ type in the color space and $\overline{3} \otimes 3$ type in the flavor space, and differs from the corresponding current constructed in Ref.[12] slightly,

$$
\widetilde{J}_{\mu}(x)=\frac{1}{2}\left[Q_{j}^{T}(x) C \gamma_{5} Q_{k}(x)+Q_{k}^{T}(x) C \gamma_{5} Q_{j}(x)\right]\left[\bar{u}_{j}(x) \gamma_{\mu} C \bar{s}_{k}^{T}(x)+\bar{u}_{k}(x) \gamma_{\mu} C \bar{s}_{j}^{T}(x)\right] .
$$

The attractive interaction induced by one-gluon exchange favors formation of the color antitriplet diquark state $Q_{j}^{T}(x) C \gamma_{\mu} Q_{k}(x)-Q_{k}^{T}(x) C \gamma_{\mu} Q_{j}(x)$, while the repulsive interaction induced by onegluon exchange disfavors formation of the color sextet diquark state $Q_{j}^{T}(x) C \gamma_{5} Q_{k}(x)+Q_{k}^{T}(x) C \gamma_{5} Q_{j}(x)$. If there really exists a doubly charmed tetraquark state $\widetilde{Z}_{Q Q}$, which couples potentially to the current $\widetilde{J}_{\mu}(x)$, then the tetraquark state $\widetilde{Z}_{Q Q}$ should have much larger mass than the corresponding tetraquark state $Z_{Q Q}$. As the color magnetic interaction $-\sum_{i<j} C_{i j} \lambda_{i} \cdot \lambda_{j} \sigma_{i} \cdot \sigma_{j}$ leads to mixing between the tetraquark states $Z_{Q Q}$ and $\widetilde{Z}_{Q Q}$, where the $\lambda_{i}$ and $\sigma_{i}$ denote the Gell-Mann matrices and Pauli matrices, respectively [1, 7]. Some $6 \otimes \overline{6}$ type components in the color space can lead to larger predicted tetraquark mass than the $M_{Z}$, for example, if we take the replacement,

$$
J_{\mu}(x) \rightarrow J_{\mu}(x) \cos \theta+\widetilde{J}_{\mu}(x) \sin \theta,
$$

we expect to obtain a tetraquark mass $M$ with the value $M_{Z}<M<M_{\widetilde{Z}}$. The conclusion survives for the current $\eta_{\mu}(x)$. However, in Ref.[12], M. L. Du et al obtain degenerate masses for the $Z_{Q Q}$ and $\widetilde{Z}_{Q Q}$ based on the QCD sum rules. This subject needs to be further studied.

In the following, we briefly outline the operator product expansion for the correlation functions $\Pi_{\mu \nu}^{J}(p)$ and $\Pi_{\mu \nu}^{\eta}(p)$ in perturbative QCD. We contract the $u, d, s$ and $Q$ quark fields in the 
correlation functions $\Pi_{\mu \nu}^{J}(p)$ and $\Pi_{\mu \nu}^{\eta}(p)$ with Wick theorem, and obtain the results:

$$
\begin{aligned}
\Pi_{\mu \nu}^{J}(p)= & -2 i \varepsilon^{i j k} \varepsilon^{i m n} \varepsilon^{i^{\prime} j^{\prime} k^{\prime}} \varepsilon^{i^{\prime} m^{\prime} n^{\prime}} \int d^{4} x e^{i p \cdot x} \\
& \operatorname{Tr}\left[\gamma_{\mu} S_{Q}^{k k^{\prime}}(x) \gamma_{\nu} C S_{Q}^{T j j^{\prime}}(x) C\right] \operatorname{Tr}\left[\gamma_{5} U^{m^{\prime} m}(-x) \gamma_{5} C S^{T n^{\prime} n}(-x) C\right], \\
\Pi_{\mu \nu}^{\eta}(p)= & -2 i \varepsilon^{i j k} \varepsilon^{i m n} \varepsilon^{i^{\prime} j^{\prime} k^{\prime}} \varepsilon^{i^{\prime} m^{\prime} n^{\prime}} \int d^{4} x e^{i p \cdot x} \\
& \operatorname{Tr}\left[\gamma_{\mu} S_{Q}^{k k^{\prime}}(x) \gamma_{\nu} C S_{Q}^{T j j^{\prime}}(x) C\right] \operatorname{Tr}\left[\gamma_{5} U^{m^{\prime} m}(-x) \gamma_{5} C D^{T n^{\prime} n}(-x) C\right],
\end{aligned}
$$

where the $U^{i j}(x), D^{i j}(x), S^{i j}(x)$ and $S_{Q}^{i j}(x)$ are the full $u, d, s$ and $Q$ quark propagators, respectively [21, 30],

$$
\begin{aligned}
& U / D_{i j}(x)=\frac{i \delta_{i j} \not \not}{2 \pi^{2} x^{4}}-\frac{\delta_{i j}\langle\bar{q} q\rangle}{12}-\frac{\delta_{i j} x^{2}\left\langle\bar{q} g_{s} \sigma G q\right\rangle}{192}-\frac{i g_{s} G_{\alpha \beta}^{a} t_{i j}^{a}\left(\not \not \sigma^{\alpha \beta}+\sigma^{\alpha \beta} \not x\right)}{32 \pi^{2} x^{2}} \\
& -\frac{1}{8}\left\langle\bar{q}_{j} \sigma^{\mu \nu} q_{i}\right\rangle \sigma_{\mu \nu}+\cdots,
\end{aligned}
$$

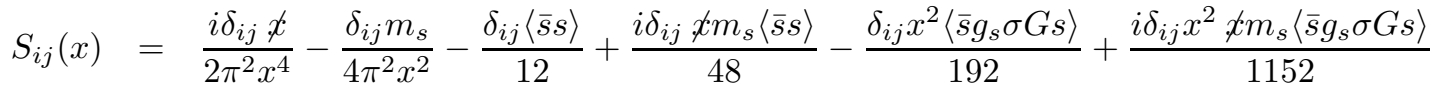

$$
\begin{aligned}
& -\frac{i g_{s} G_{\alpha \beta}^{a} t_{i j}^{a}\left(\not \partial \sigma^{\alpha \beta}+\sigma^{\alpha \beta} \not x\right)}{32 \pi^{2} x^{2}}-\frac{1}{8}\left\langle\bar{s}_{j} \sigma^{\mu \nu} s_{i}\right\rangle \sigma_{\mu \nu}+\cdots, \\
& S_{Q}^{i j}(x)=\frac{i}{(2 \pi)^{4}} \int d^{4} k e^{-i k \cdot x}\left\{\frac{\delta_{i j}}{\not k-m_{Q}}-\frac{g_{s} G_{\alpha \beta}^{n} t_{i j}^{n}}{4} \frac{\sigma^{\alpha \beta}\left(\not k+m_{Q}\right)+\left(\not k+m_{Q}\right) \sigma^{\alpha \beta}}{\left(k^{2}-m_{Q}^{2}\right)^{2}}\right. \\
& \left.-\frac{g_{s}^{2}\left(t^{a} t^{b}\right)_{i j} G_{\alpha \beta}^{a} G_{\mu \nu}^{b}\left(f^{\alpha \beta \mu \nu}+f^{\alpha \mu \beta \nu}+f^{\alpha \mu \nu \beta}\right)}{4\left(k^{2}-m_{Q}^{2}\right)^{5}}+\cdots\right\} \\
& f^{\lambda \alpha \beta}=\left(\not k+m_{Q}\right) \gamma^{\lambda}\left(\not k+m_{Q}\right) \gamma^{\alpha}\left(\not k+m_{Q}\right) \gamma^{\beta}\left(\not k+m_{Q}\right), \\
& f^{\alpha \beta \mu \nu}=\left(\not k+m_{Q}\right) \gamma^{\alpha}\left(\not k+m_{Q}\right) \gamma^{\beta}\left(\not k+m_{Q}\right) \gamma^{\mu}\left(\not k+m_{Q}\right) \gamma^{\nu}\left(\not k+m_{Q}\right) \text {. }
\end{aligned}
$$

Then we compute the integrals both in coordinate space and in momentum space, and obtain the correlation functions $\Pi_{J / \eta}\left(p^{2}\right)$ at the quark level, therefore the QCD spectral densities through dispersion relation.

$$
\lim _{\epsilon \rightarrow 0} \frac{\operatorname{Im}_{J / \eta}(s+i \epsilon)}{\pi}=\rho_{J / \eta}(s) .
$$

In Eqs.(14-15), we retain the terms $\left\langle\bar{q}_{j} \sigma_{\mu \nu} q_{i}\right\rangle$ and $\left\langle\bar{s}_{j} \sigma_{\mu \nu} s_{i}\right\rangle$ come from the Fierz re-ordering of the $\left\langle q_{i} \bar{q}_{j}\right\rangle$ and $\left\langle s_{i} \bar{s}_{j}\right\rangle$ to absorb the gluons emitted from other quark lines to form $\left\langle\bar{q}_{j} g_{s} G_{\alpha \beta}^{a} t_{m n}^{a} \sigma_{\mu \nu} q_{i}\right\rangle$ and $\left\langle\bar{s}_{j} g_{s} G_{\alpha \beta}^{a} t_{m n}^{a} \sigma_{\mu \nu} s_{i}\right\rangle$ to extract the mixed condensates $\left\langle\bar{q} g_{s} \sigma G q\right\rangle$ and $\left\langle\bar{s} g_{s} \sigma G s\right\rangle$, respectively. In this article, we carry out the operator product expansion to the vacuum condensates up to dimension-10, and take into account the vacuum condensates which are vacuum expectations of the operators of the orders $\mathcal{O}\left(\alpha_{s}^{k}\right)$ with $k \leq 1$ in a consistent way [23, 24, 25, 26, 27].

Once the analytical expressions of the QCD spectral densities $\rho_{J / \eta}(s)$ are obtained, we can take the quark-hadron duality below the continuum thresholds $s_{0}$ and perform Borel transform with respect to the variable $P^{2}=-p^{2}$ to obtain the following QCD sum rules,

$$
\lambda_{Z}^{2} \exp \left(-\frac{M_{Z}^{2}}{T^{2}}\right)=\int_{4 m_{Q}^{2}}^{s_{0}} d s \rho_{J / \eta}(s) \exp \left(-\frac{s}{T^{2}}\right)
$$


where

$$
\begin{aligned}
& \rho_{J}(s)=\rho_{0}(s)+\rho_{3}(s)+\rho_{4}(s)+\rho_{5}(s)+\rho_{6}(s)+\rho_{8}(s)+\rho_{10}(s), \\
& \rho_{\eta}(s)=\left.\rho_{J}(s)\right|_{m_{s} \rightarrow 0,\langle\bar{s} s\rangle \rightarrow\langle\bar{q} q\rangle,\left\langle\bar{s} g_{s} \sigma G s\right\rangle \rightarrow\left\langle\bar{q} g_{s} \sigma G q\right\rangle}, \\
& \rho_{0}(s)=\frac{1}{512 \pi^{6}} \int_{y_{i}}^{y_{f}} d y \int_{z_{i}}^{1-y} d z y z(1-y-z)^{2}\left(s-\bar{m}_{Q}^{2}\right)^{3}\left(5 s-\bar{m}_{Q}^{2}\right) \\
& +\frac{m_{Q}^{2}}{128 \pi^{6}} \int_{y_{i}}^{y_{f}} d y \int_{z_{i}}^{1-y} d z(1-y-z)^{2}\left(s-\bar{m}_{Q}^{2}\right)^{3}, \\
& \rho_{3}(s)=\frac{m_{s}[\langle\bar{s} s\rangle-2\langle\bar{q} q\rangle]}{32 \pi^{4}} \int_{y_{i}}^{y_{f}} d y \int_{z_{i}}^{1-y} d z y z\left(s-\bar{m}_{Q}^{2}\right)\left(3 s-\bar{m}_{Q}^{2}\right) \\
& +\frac{m_{s} m_{Q}^{2}[\langle\bar{s} s\rangle-2\langle\bar{q} q\rangle]}{16 \pi^{4}} \int_{y_{i}}^{y_{f}} d y \int_{z_{i}}^{1-y} d z\left(s-\bar{m}_{Q}^{2}\right), \\
& \rho_{4}(s)=-\frac{m_{Q}^{2}}{384 \pi^{4}}\left\langle\frac{\alpha_{s} G G}{\pi}\right\rangle \int_{y_{i}}^{y_{f}} d y \int_{z_{i}}^{1-y} d z\left(\frac{z}{y^{2}}+\frac{y}{z^{2}}\right)(1-y-z)^{2}\left(2 s-\bar{m}_{Q}^{2}\right) \\
& -\frac{m_{Q}^{4}}{384 \pi^{4}}\left\langle\frac{\alpha_{s} G G}{\pi}\right\rangle \int_{y_{i}}^{y_{f}} d y \int_{z_{i}}^{1-y} d z\left(\frac{1}{y^{3}}+\frac{1}{z^{3}}\right)(1-y-z)^{2} \\
& +\frac{m_{Q}^{2}}{128 \pi^{4}}\left\langle\frac{\alpha_{s} G G}{\pi}\right\rangle \int_{y_{i}}^{y_{f}} d y \int_{z_{i}}^{1-y} d z\left(\frac{1}{y^{2}}+\frac{1}{z^{2}}\right)(1-y-z)^{2}\left(s-\bar{m}_{Q}^{2}\right) \\
& -\frac{1}{1536 \pi^{4}}\left\langle\frac{\alpha_{s} G G}{\pi}\right\rangle \int_{y_{i}}^{y_{f}} d y \int_{z_{i}}^{1-y} d z(1-y-z)^{2}\left(s-\bar{m}_{Q}^{2}\right)\left(5 s-3 \bar{m}_{Q}^{2}\right) \\
& +\frac{1}{256 \pi^{4}}\left\langle\frac{\alpha_{s} G G}{\pi}\right\rangle \int_{y_{i}}^{y_{f}} d y \int_{z_{i}}^{1-y} d z y z\left(s-\bar{m}_{Q}^{2}\right)\left(3 s-\bar{m}_{Q}^{2}\right) \\
& +\frac{m_{Q}^{2}}{128 \pi^{4}}\left\langle\frac{\alpha_{s} G G}{\pi}\right\rangle \int_{y_{i}}^{y_{f}} d y \int_{z_{i}}^{1-y} d z\left(s-\bar{m}_{Q}^{2}\right) \\
& \rho_{5}(s)=\frac{m_{s}\left[3\left\langle\bar{q} g_{s} \sigma G q\right\rangle-\left\langle\bar{s} g_{s} \sigma G s\right\rangle\right]}{48 \pi^{4}} \int_{y_{i}}^{y_{f}} d y y(1-y) s, \\
& \rho_{6}(s)=\frac{\langle\bar{q} q\rangle\langle\bar{s} s\rangle}{3 \pi^{2}} \int_{y_{i}}^{y_{f}} d y y(1-y) s \\
& \rho_{8}(s)=-\frac{\langle\bar{s} s\rangle\left\langle\bar{q} g_{s} \sigma G q\right\rangle+\langle\bar{q} q\rangle\left\langle\bar{s} g_{s} \sigma G s\right\rangle}{24 \pi^{2}} \int_{y_{i}}^{y_{f}} d y y(1-y)\left[3+\left(4 s+\frac{2 s^{2}}{T^{2}}\right) \delta\left(s-\widetilde{m}_{Q}^{2}\right)\right], \\
& \rho_{10}(s)=\frac{\left\langle\bar{q} g_{s} \sigma G q\right\rangle\left\langle\bar{s} g_{s} \sigma G s\right\rangle}{48 \pi^{2}} \int_{y_{i}}^{y_{f}} d y y(1-y)\left(\frac{s}{T^{2}}+\frac{2 s^{2}}{T^{4}}+\frac{s^{3}}{T^{6}}\right) \delta\left(s-\widetilde{m}_{Q}^{2}\right) \\
& -\frac{11\left\langle\bar{q} g_{s} \sigma G q\right\rangle\left\langle\bar{s} g_{s} \sigma G s\right\rangle}{6912 \pi^{2}} \int_{y_{i}}^{y_{f}} d y\left(1+\frac{s}{2 T^{2}}\right) \delta\left(s-\widetilde{m}_{Q}^{2}\right)
\end{aligned}
$$


$y_{f}=\frac{1+\sqrt{1-4 m_{Q}^{2} / s}}{2}, y_{i}=\frac{1-\sqrt{1-4 m_{Q}^{2} / s}}{2}, z_{i}=\frac{y m_{Q}^{2}}{y s-m_{Q}^{2}}, \bar{m}_{Q}^{2}=\frac{(y+z) m_{Q}^{2}}{y z}, \widetilde{m}_{Q}^{2}=\frac{m_{Q}^{2}}{y(1-y)}, \int_{y_{i}}^{y_{f}} d y \rightarrow \int_{0}^{1} d y$, $\int_{z_{i}}^{1-y} d z \rightarrow \int_{0}^{1-y} d z$ when the $\delta$ functions $\delta\left(s-\bar{m}_{Q}^{2}\right)$ and $\delta\left(s-\widetilde{m}_{Q}^{2}\right)$ appear.

We derive Eq.(19) with respect to $\tau=\frac{1}{T^{2}}$, then eliminate the pole residues $\lambda_{Z}$ to obtain the QCD sum rules for the masses,

$$
M_{Z}^{2}=\frac{-\frac{d}{d \tau} \int_{4 m_{Q}^{2}}^{s_{0}} d s \rho_{J / \eta}(s) e^{-\tau s}}{\int_{4 m_{Q}^{2}}^{s_{0}} d s \rho_{J / \eta}(s) e^{-\tau s}}
$$

\section{Numerical results and discussions}

We take the standard values of the vacuum condensates $\langle\bar{q} q\rangle=-(0.24 \pm 0.01 \mathrm{GeV})^{3},\left\langle\bar{q} g_{s} \sigma G q\right\rangle=$ $m_{0}^{2}\langle\bar{q} q\rangle, m_{0}^{2}=(0.8 \pm 0.1) \mathrm{GeV}^{2},\langle\bar{s} s\rangle=(0.8 \pm 0.1)\langle\bar{q} q\rangle,\left\langle\bar{s} g_{s} \sigma G s\right\rangle=m_{0}^{2}\langle\bar{s} s\rangle,\left\langle\frac{\alpha_{s} G G}{\pi}\right\rangle=(0.33 \mathrm{GeV})^{4}$ at the energy scale $\mu=1 \mathrm{GeV}\left[20,21\right.$, 31], and choose the $\overline{M S}$ masses $m_{c}\left(m_{c}\right)=(1.275 \pm 0.025) \mathrm{GeV}$, $m_{b}\left(m_{b}\right)=(4.18 \pm 0.03) \mathrm{GeV}, m_{s}(\mu=2 \mathrm{GeV})=(0.095 \pm 0.005) \mathrm{GeV}$ from the Particle Data Group [32. Furthermore, we take into account the energy-scale dependence of the input parameters,

$$
\begin{aligned}
\langle\bar{q} q\rangle(\mu) & =\langle\bar{q} q\rangle(Q)\left[\frac{\alpha_{s}(Q)}{\alpha_{s}(\mu)}\right]^{\frac{4}{9}}, \\
\langle\bar{s} s\rangle(\mu) & =\langle\bar{s} s\rangle(Q)\left[\frac{\alpha_{s}(Q)}{\alpha_{s}(\mu)}\right]^{\frac{4}{9}}, \\
\left\langle\bar{q} g_{s} \sigma G q\right\rangle(\mu) & =\left\langle\bar{q} g_{s} \sigma G q\right\rangle(Q)\left[\frac{\alpha_{s}(Q)}{\alpha_{s}(\mu)}\right]^{\frac{2}{27}}, \\
\left\langle\bar{s} g_{s} \sigma G s\right\rangle(\mu) & =\left\langle\bar{s} g_{s} \sigma G s\right\rangle(Q)\left[\frac{\alpha_{s}(Q)}{\alpha_{s}(\mu)}\right]^{\frac{2}{27}}, \\
m_{c}(\mu) & =m_{c}\left(m_{c}\right)\left[\frac{\alpha_{s}(\mu)}{\alpha_{s}\left(m_{c}\right)}\right]^{\frac{12}{25}}, \\
m_{b}(\mu) & =m_{b}\left(m_{b}\right)\left[\frac{\alpha_{s}(\mu)}{\alpha_{s}\left(m_{b}\right)}\right]^{\frac{12}{23}}, \\
m_{s}(\mu) & =m_{s}(2 \mathrm{GeV})\left[\frac{\alpha_{s}(\mu)}{\alpha_{s}(2 \mathrm{GeV})}\right]^{\frac{4}{9}}, \\
\alpha_{s}(\mu) & =\frac{1}{b_{0} t}\left[1-\frac{b_{1}}{b_{0}^{2}} \frac{\log t}{t}+\frac{b_{1}^{2}\left(\log ^{2} t-\log t-1\right)+b_{0} b_{2}}{b_{0}^{4} t^{2}},\right.
\end{aligned}
$$

where $t=\log \frac{\mu^{2}}{\Lambda^{2}}, b_{0}=\frac{33-2 n_{f}}{12 \pi}, b_{1}=\frac{153-19 n_{f}}{24 \pi^{2}}, b_{2}=\frac{2857-\frac{5033}{9} n_{f}+\frac{325}{27} n_{f}^{2}}{128 \pi^{3}}, \Lambda=213 \mathrm{MeV}, 296 \mathrm{MeV}$ and $339 \mathrm{MeV}$ for the flavors $n_{f}=5,4$ and 3, respectively [32], and evolve all the input parameters to the optimal energy scales $\mu$ to extract the masses of the $Z_{Q Q}$.

In Refs. 23, 24, 25, 26, 27, we study the acceptable energy scales of the QCD spectral densities for the hidden-charm (hidden-bottom) tetraquark states and molecular states in the QCD sum rules in details for the first time, and suggest an energy scale formula $\mu=\sqrt{M_{X / Y / Z}^{2}-\left(2 \mathbb{M}_{Q}\right)^{2}}$ to determine the optimal energy scales, which enhances the pole contributions remarkably and works well. The energy scale formula also works well in studying the hidden-charm pentaquark states [33. We can assign the $Z_{c}(3900)$ and $Z_{b}(10610)$ to be the axialvector tetraquark states with the 
quark constituents $c u \bar{c} \bar{d}$ and $b u \bar{b} \bar{d}$ respectively, and choose the currents,

$$
J_{\mu}^{Q \bar{Q}}(x)=\frac{\varepsilon^{i j k} \varepsilon^{i m n}}{\sqrt{2}}\left\{u_{j}^{T}(x) C \gamma_{5} Q_{k}(x) \bar{d}_{m}(x) \gamma_{\mu} C \bar{Q}_{n}^{T}(x)-u_{j}^{T}(x) C \gamma_{\mu} Q_{k}(x) \bar{d}_{m}(x) \gamma_{5} C \bar{Q}_{n}^{T}(x)\right\},
$$

with $Q=c, b$ to study them with the QCD sum rules 23, 26. If we take the updated values of the effective heavy quark masses $\mathbb{M}_{c}=1.82 \mathrm{GeV}$ and $\mathbb{M}_{b}=5.17 \mathrm{GeV}$ [34], the optimal energy scales of the QCD spectral densities of the $Z_{c}(3900)$ and $Z_{b}(10610)$ are $\mu=1.4 \mathrm{GeV}$ and $2.4 \mathrm{GeV}$, respectively.

There are no experimental candidates for the doubly heavy tetraquark states. Firstly, we suppose that the ground state $C \gamma_{\mu} \otimes \gamma_{5} C$ type axialvector tetraquark states $Q Q \bar{u} \bar{d}$ and $Q u \bar{Q} \bar{d}$ have degenerate masses, and study the masses of the ground state axialvector tetraquark states $Q Q \bar{u} \bar{d}$ at the same energy scales of the QCD spectral densities as the ones for the ground state axialvector tetraquark states $Q u \bar{Q} \bar{d}$. In Fig.1, we plot the predicted masses of the $Z_{c c \bar{u} \bar{d}}\left(Z_{b b \bar{u} \bar{d}}\right)$ and $Z_{c}(3900)$ $\left(Z_{b}(10610)\right)$ with variations of the Borel parameter $T^{2}$ for the continuum threshold parameter $\sqrt{s_{0}}=4.4 \mathrm{GeV}\left(s_{0}^{2}=124 \mathrm{GeV}^{2}\right)$ and the energy scale $\mu=1.4 \mathrm{GeV}(\mu=2.4 \mathrm{GeV})[23$, 26, 34]. From the figure, we can see that the experimental values of the masses of the $Z_{c}(3900)$ and $Z_{b}(10610)$ can be well reproduced, there appear platforms for the masses of the $Q Q \bar{u} \bar{d}$ tetraquark states, which lie slightly below the corresponding masses of the $Z_{c}(3900)$ and $Z_{b}(10610)$, respectively. If we choose the Borel windows as $T^{2}=(2.6-3.0) \mathrm{GeV}^{2}$ and $(6.9-7.7) \mathrm{GeV}^{2}$ for the tetraquark states $c c \bar{u} \bar{d}$ and $b b \bar{u} \bar{d}$, respectively, the pole contributions are $(44-58) \%$ and $(44-56) \%$, respectively, it is reliable to extract the masses. Furthermore, the continuum threshold parameters $s_{0}$ satisfy the relation $\sqrt{s_{0}}-M_{c c \bar{u} \bar{d}}=0.55 \mathrm{GeV}$ and $\sqrt{s_{0}}-M_{b b \bar{u} \bar{d}}=0.62 \mathrm{GeV}$, respectively, which are consistent with our naive expectation that the mass gaps of the ground states and the first radial excited states of the tetraquark states are about $(0.5-0.6) \mathrm{GeV}[35$, 36]. The energy scales $\mu=1.4 \mathrm{GeV}$ and $2.4 \mathrm{GeV}$ work well.

In Ref. [8, Karliner and Rosner obtain the masses $M=3.882 \mathrm{GeV}$ and $10.389 \mathrm{GeV}$ for the $C \gamma_{\mu} \otimes \gamma_{5} C$ type axialvector tetraquark states $c c \bar{u} \bar{d}$ and $b b \bar{u} \bar{d}$ respectively based on a simple potential quark model, which can reproduce the mass of the doubly charmed baryon state $\Xi_{c c}^{++}$. In Ref. [16], Eichten and Quigg obtain the masses $M=3.978 \mathrm{GeV}$ and $10.468 \mathrm{GeV}$ for the $C \gamma_{\mu} \otimes \gamma_{5} C$ type axialvector tetraquark states $c c \bar{u} \bar{d}$ and $b b \bar{u} \bar{d}$ respectively based on the heavy quark symmetry, where the mass of the doubly charmed baryon state $\Xi_{c c}^{++}$is taken as input parameter in the charm sector, while in the bottom sector, there are no experimental candidates for the baryon states $\Xi_{b b}^{0}$ and $\Xi_{b b}^{-}$. From Fig.1, we can see that if we take the same parameters, such as the energy scales, continuum threshold parameters, etc, in the charm sector, the predicted mass $M_{c c \bar{u} \bar{d}}=3.85 \mathrm{GeV}$ is slightly smaller than the value $3.882 \mathrm{GeV}$ from a simple potential quark model $[8$ and much smaller than the value $3.978 \mathrm{GeV}$ from the heavy quark symmetry [16], in the bottom sector, the predicted mass $M_{b b \bar{u} \bar{d}}=10.52 \mathrm{GeV}$ is much larger than the value $10.389 \mathrm{GeV}$ from a simple potential quark model [8] and slightly larger than the value $10.468 \mathrm{GeV}$ from the heavy quark symmetry [16].

Now we revisit the subject of how to choose the energy scales of the QCD spectral densities. In calculation, we neglect the perturbative corrections to the currents $J / \eta_{\alpha}(x)$, which can be taken into account in the leading logarithmic approximation through an anomalous dimension factor, $\left[\frac{\alpha_{s}\left(\mu_{0}\right)}{\alpha_{s}(\mu)}\right]^{\gamma_{J}}$, the $\gamma_{J}$ are the anomalous dimension of the interpolating currents $J / \eta(x)$,

$$
\begin{aligned}
\left\langle 0\left|J / \eta_{\alpha}(0 ; \mu)\right| Z_{Q Q}(p)\right\rangle & =\left[\frac{\alpha_{s}\left(\mu_{0}\right)}{\alpha_{s}(\mu)}\right]^{\gamma_{J}}\left\langle 0\left|J / \eta_{\alpha}\left(0 ; \mu_{0}\right)\right| Z_{Q Q}(p)\right\rangle \\
& =\left[\frac{\alpha_{s}\left(\mu_{0}\right)}{\alpha_{s}(\mu)}\right]^{\gamma_{J}} \lambda_{Z}\left(\mu_{0}\right) \varepsilon_{\alpha}=\lambda_{Z}(\mu) \varepsilon_{\alpha} .
\end{aligned}
$$

The pole residues $\lambda_{Z}(\mu)=\left[\frac{\alpha_{s}\left(\mu_{0}\right)}{\alpha_{s}(\mu)}\right]^{\gamma_{J}} \lambda_{Z}\left(\mu_{0}\right)$ are energy scale dependent quantities, at the leading order approximation, we can set $\gamma_{J}=0$. 

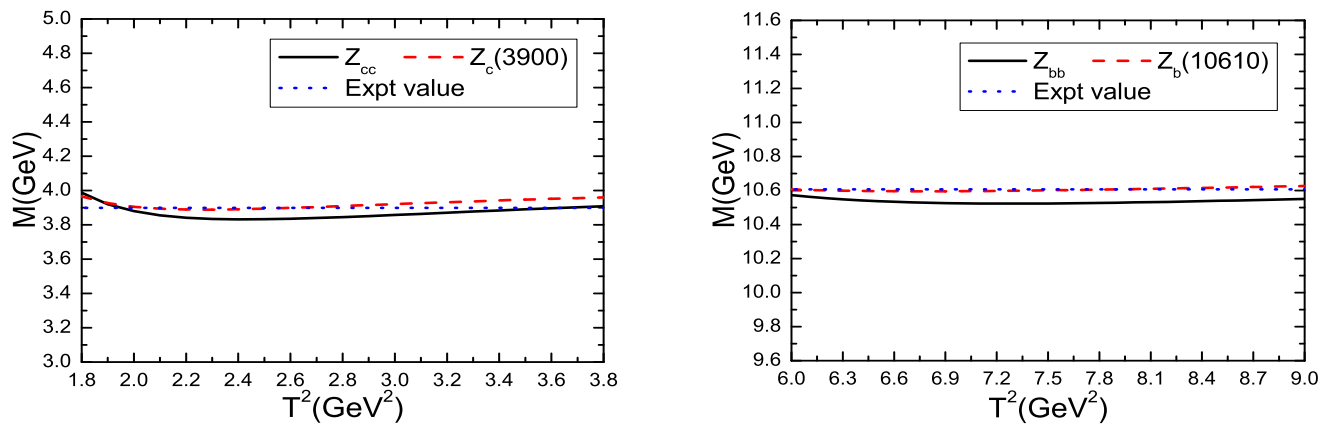

Figure 1: The masses of the $Z_{c c \bar{u} \bar{d}}, Z_{c}(3900), Z_{b b \bar{u} \bar{d}}$ and $Z_{b}(10610)$ with variations of the Borel parameter $T^{2}$, where the Expt value denotes the experimental values of the masses $M_{Z_{c}(3900)}$ and $M_{Z_{b}(10610)}$.

At the QCD side, the correlation functions $\Pi\left(p^{2}\right)$ can be written as

$$
\Pi\left(p^{2}\right)=\int_{4 m_{Q}^{2}(\mu)}^{s_{0}} d s \frac{\rho_{J / \eta}(s, \mu)}{s-p^{2}}+\int_{s_{0}}^{\infty} d s \frac{\rho_{J / \eta}(s, \mu)}{s-p^{2}}
$$

through dispersion relation, and they are energy scale independent according to the approximation $\gamma_{J}=0$ or $\left[\frac{\alpha_{s}\left(\mu_{0}\right)}{\alpha_{s}(\mu)}\right]^{\gamma_{J}}=1$

$$
\frac{d}{d \mu} \Pi\left(p^{2}\right)=0
$$

which does not mean the pole contributions are energy scale independent,

$$
\frac{d}{d \mu} \int_{4 m_{Q}^{2}(\mu)}^{s_{0}} d s \frac{\rho_{J / \eta}(s, \mu)}{s-p^{2}} \rightarrow 0
$$

due to the following two reasons inherited from the QCD sum rules: (I) Perturbative corrections are neglected, the higher dimensional vacuum condensates are factorized into lower dimensional ones therefore the energy scale dependence of the higher dimensional vacuum condensates is modified; (II) Truncations $s_{0}$ set in, the correlation between the threshold $4 m_{Q}^{2}(\mu)$ and continuum threshold $s_{0}$ is unknown, the quark-hadron duality is just an assumption. Even if the anomalous dimensions $\gamma_{J}$ are neglected, the pole residues $\lambda_{Z}$ acquire energy scale dependence through the QCD side of the QCD sum rules, which does not mean that we cannot extract reliable information of bound states.

In the article, we study the doubly heavy tetraquark states, the two heavy quarks form an axialvector doubly heavy diquark state in color antitriplet, then the axialvector doubly heavy diquark state serves as a static well potential and combines with a light antidiquark state in color triplet to form a compact tetraquark state. Such a tetraquark system is also characterized by the effective heavy quark mass $\mathbb{M}_{Q}$ and the virtuality $V=\sqrt{M_{X / Y / Z}^{2}-\left(2 \mathbb{M}_{Q}\right)^{2}}$ (or bound energy not as robust) $24,25,26$. We obtain the energy scale formula by setting the energy scale $\mu=V$. It is not necessary for the effective heavy quark masses $\mathbb{M}_{Q}$ in the doubly heavy tetraquark states to have the same values as the ones in the hidden-charm and hidden-bottom tetraquark states. In calculations, we observe that if we choose a slightly larger value $\mathbb{M}_{c}=1.84 \mathrm{GeV}$, the criteria of the QCD sum rules (pole dominance at the hadron side and convergence of the operator product expansion at the QCD side) can be satisfied more easily, furthermore, other doubly charmed 


\begin{tabular}{|c|c|c|c|c|c|c|}
\hline \hline & $T^{2}\left(\mathrm{GeV}^{2}\right)$ & $\sqrt{s_{0}}(\mathrm{GeV})$ & $\mu(\mathrm{GeV})$ & pole & $M(\mathrm{GeV})$ & $\lambda\left(\mathrm{GeV}^{5}\right)$ \\
\hline$c c \bar{u} d$ & $2.6-3.0$ & $4.45 \pm 0.10$ & 1.3 & $(39-63) \%$ & $3.90 \pm 0.09$ & $(2.64 \pm 0.42) \times 10^{-2}$ \\
\hline$c c \bar{u} \bar{s}$ & $2.6-3.0$ & $4.50 \pm 0.10$ & 1.3 & $(41-64) \%$ & $3.95 \pm 0.08$ & $(2.88 \pm 0.46) \times 10^{-2}$ \\
\hline$b b \bar{u} d$ & $6.9-7.7$ & $11.14 \pm 0.10$ & 2.4 & $(41-60) \%$ & $10.52 \pm 0.08$ & $(1.30 \pm 0.20) \times 10^{-1}$ \\
\hline$b b \bar{u} \bar{s}$ & $6.8-7.6$ & $11.15 \pm 0.10$ & 2.4 & $(41-61) \%$ & $10.55 \pm 0.08$ & $(1.33 \pm 0.20) \times 10^{-1}$ \\
\hline$c c \bar{u} d$ & $2.6-3.0$ & $4.40 \pm 0.10$ & 1.4 & $(39-62) \%$ & $3.85 \pm 0.09$ & $(2.60 \pm 0.42) \times 10^{-2}$ \\
\hline \hline
\end{tabular}

Table 1: The Borel parameters (Borel windows), continuum threshold parameters, ideal energy scales, pole contributions, masses and pole residues for the doubly heavy tetraquark states.

tetraquark states, such as the $C \gamma_{\mu} \otimes \gamma_{\nu} C$-type scalar, axialvector, tensor and vector tetraquark states, can be described in the same routine [37. While in the bottom sector, a slightly smaller value $\mathbb{M}_{b}=5.12 \mathrm{GeV}$ does the work. In this article, we choose the values $\mathbb{M}_{c}=1.84 \mathrm{GeV}$ and $\mathbb{M}_{b}=5.12 \mathrm{GeV}$, and take into account the $S U(3)$ breaking effect $m_{s}(\mu)$ by subtracting the $m_{s}(\mu)$ from the virtuality $V, \mu_{k}=V_{k}=\sqrt{M_{X / Y / Z}^{2}-\left(2 \mathbb{M}_{Q}\right)^{2}}-k m_{s}\left(\mu_{k}\right)$, where the numbers of the strange antiquark $\bar{s}$ in the doubly heavy tetraquark states are $k=0,1$. We cannot obtain energy scale independent QCD sum rules, but we have an energy scale formula to determine the energy scales consistently.

In this article, we take the continuum threshold parameters as $\sqrt{s_{0}}=M_{Z}+(0.4 \sim 0.7) \mathrm{GeV}$, and vary the parameters $\sqrt{s_{0}}$ to find the optimal Borel parameters $T^{2}$ to satisfy the following four criteria:

1. Pole dominance on the phenomenological side;

2. Convergence of the operator product expansion;

3. Appearance of the Borel platforms;

4. Satisfying the energy scale formula.

The resulting Borel parameters or Borel windows $T^{2}$, continuum threshold parameters $s_{0}$, optimal energy scales of the QCD spectral densities, pole contributions of the ground states are shown explicitly in Table 1. From Table 1, we can see that the pole dominance can be well satisfied. In Table 1, we also present the results where the same parameters as the ones in the QCD sum rules for the $Z_{c}(3900)$ are chosen, see the last line.

In Fig.2, we plot the contributions of the vacuum condensates in the operator product expansion with variations of the Borel parameter $T^{2}$ at much larger ranges than the Borel windows for the central values of the threshold parameters shown in Table 1. From the figure, we can see that although the dominant contributions do not come from the perturbative terms, the contributions of the vacuum condensates of dimensions $n=6$ and 8 are very large, but the contributions of the vacuum condensates of dimensions $6,8,10$ have the hierarchy $D_{6}>\left|D_{8}\right| \gg D_{10}$ or $D_{6} \gg\left|D_{8}\right| \gg$ $D_{10}$ in the Borel windows, the operator product expansion is convergent.

In the QCD sum rules for the tetraquark states and pentaquark states, the higher dimension vacuum condensates are always factorized to lower dimension vacuum condensates with vacuum saturation, factorization works well in large $N_{c}$ limit [20. In reality, $N_{c}=3$, some (not much) ambiguities maybe come from the vacuum saturation assumption. We choose universal values for the $\mathbb{M}_{Q}$, analogous pole contributions $((40-60) \%)$ and analogous criteria for the convergence of the operator product expansion $\left(D_{6}>\left|D_{8}\right| \gg D_{10}\right.$ or $\left.D_{6} \gg\left|D_{8}\right| \gg D_{10}\right)$, the ambiguities are partially absorbed into the effective masses $\mathbb{M}_{Q}$. In previous works, we observed that vacuum saturation assumption works well for all the hidden-charm (hidden-bottom) tetraquark (molecular) states and hidden-charm pentaquark states [23, 24, 25, 26, 27, 33, the ambiguities originate from the vacuum saturation cannot impair the predictive ability remarkably.

We take into account all uncertainties of the input parameters, and obtain the values of the masses and pole residues of the $Z_{Q Q}$, which are shown explicitly in Table 1 and Figs.3-4. From 

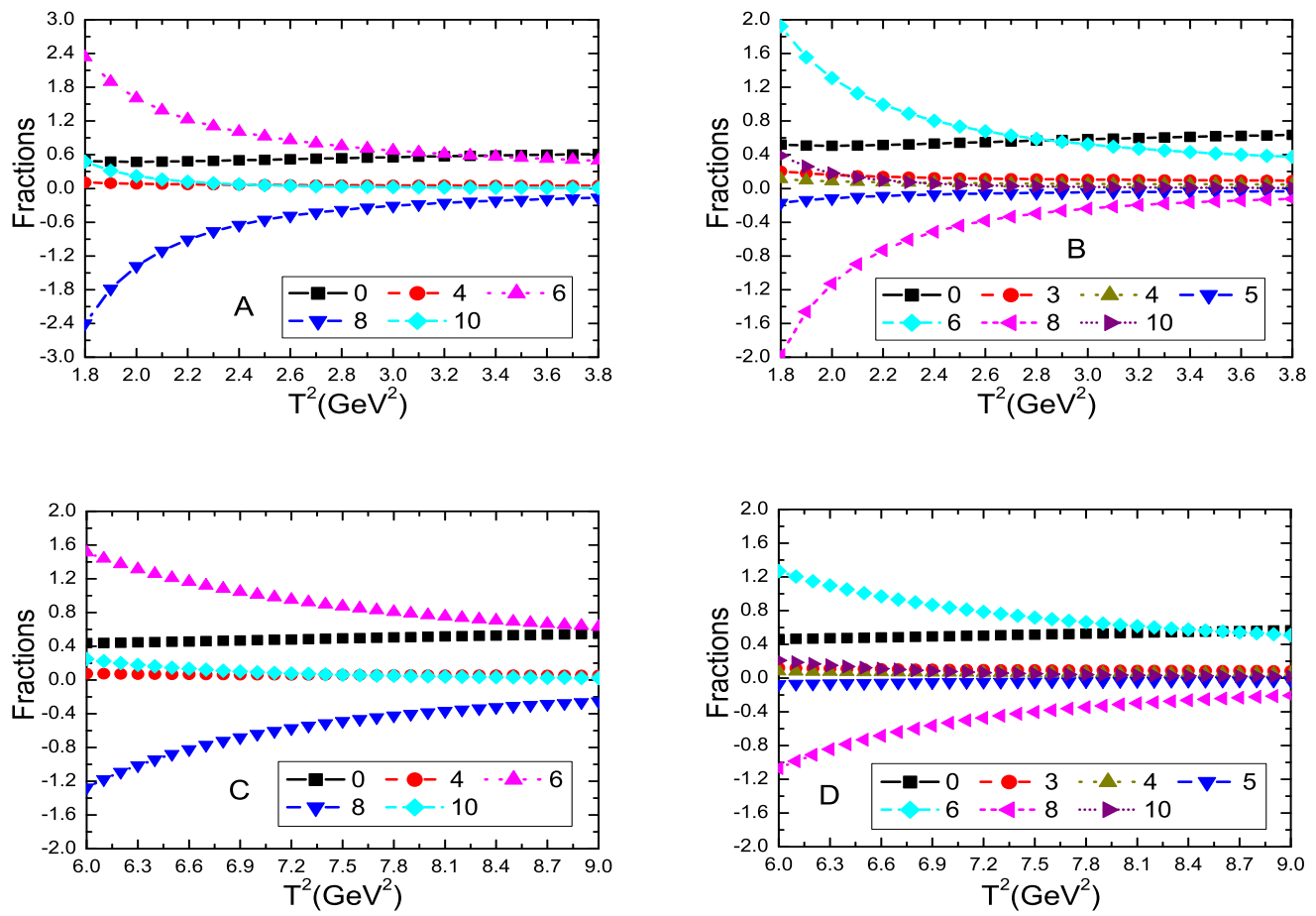

Figure 2: The contributions of different terms in the operator product expansion with variations of the Borel parameter $T^{2}$, where the $0,3,4,5,6,8$ and 10 denote the dimensions of the vacuum condensates, the $A, B, C$ and $D$ denote the tetraquark states $c c \bar{u} \bar{d}, c c \bar{u} \bar{s}, b b \bar{u} \bar{d}$ and $b b \bar{u} \bar{s}$, respectively. 

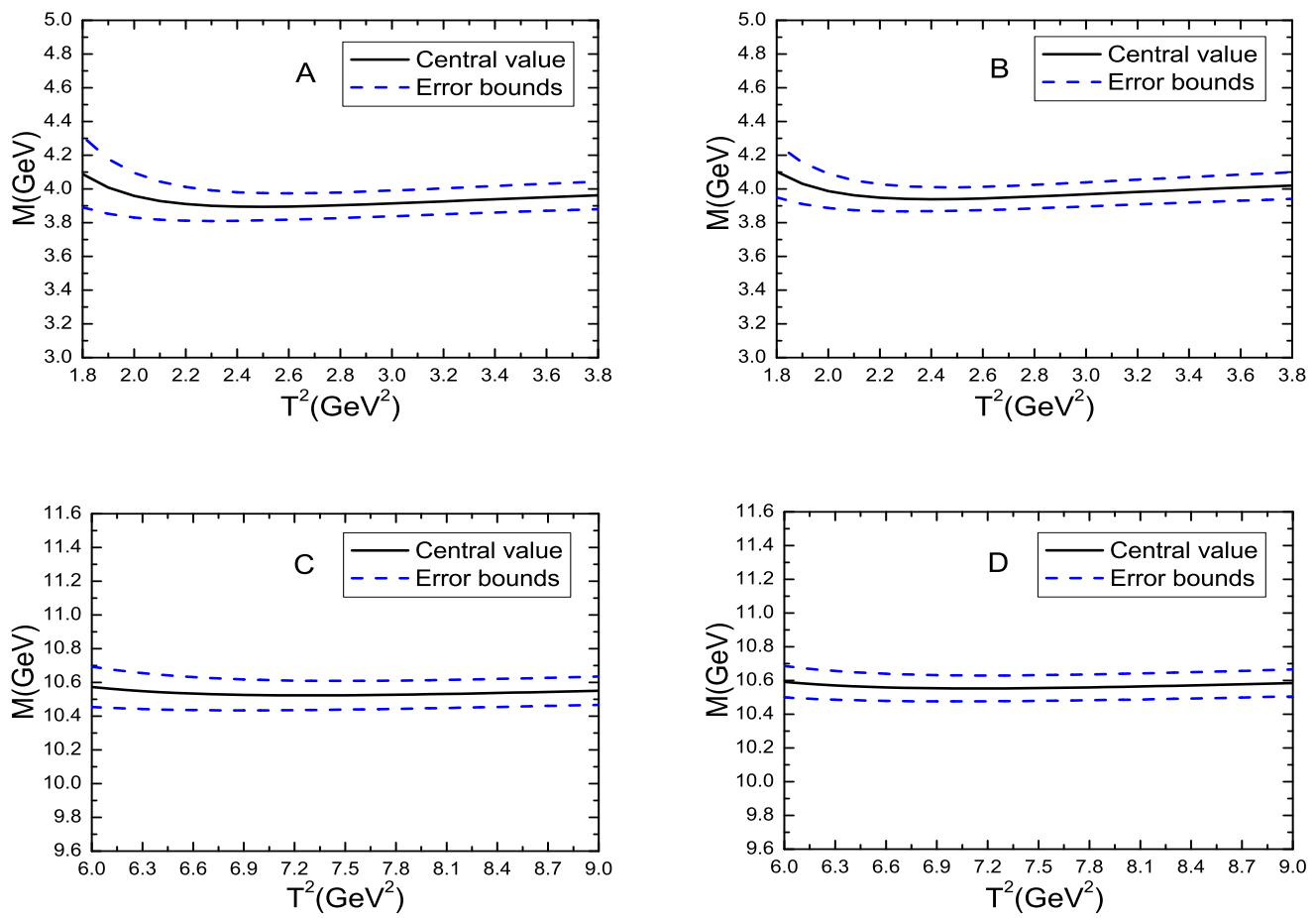

Figure 3: The masses with variations of the Borel parameter $T^{2}$, where the $A, B, C$ and $D$ denote the tetraquark states $c c \bar{u} \bar{d}, c c \bar{u} \bar{s}, b b \bar{u} \bar{d}$ and $b b \bar{u} \bar{s}$, respectively.

Figs.3-4, we can see that there appear platforms in the Borel windows shown in Table 1. Furthermore, from Table 1 , we can see that the energy scale formula $\mu_{k}=\sqrt{M_{X / Y / Z}^{2}-\left(2 \mathbb{M}_{Q}\right)^{2}}-k m_{s}\left(\mu_{k}\right)$ with $k=0,1$ is also satisfied. Moreover, from Table 1 , we can see that the Borel parameters $T=(1.6-1.7) \mathrm{GeV}$ and $(2.6-2.8) \mathrm{GeV}$ for the doubly heavy tetraquark states $Z_{c c}$ and $Z_{b b}$, respectively, which satisfy the relation $\mu=\mathcal{O}(T)$. In the regions $T=(1.6-1.7) \mathrm{GeV}$ and $(2.6-2.8) \mathrm{GeV}$ or $\mu=1.3 \mathrm{GeV}$ and $2.4 \mathrm{GeV}$, we expect to extract reliable information of bound states. Now the four criteria are all satisfied, we expect to make reliable predictions.

In this article, we have neglected the perturbative $\mathcal{O}\left(\alpha_{s}\right)$ corrections to the perturbative terms. We can estimate the effects of the perturbative $\mathcal{O}\left(\alpha_{s}\right)$ corrections by multiplying the perturbative terms by a factor 1.3 as the perturbative $\mathcal{O}\left(\alpha_{s}\right)$ corrections to the perturbative terms are usually about $30 \%$. For example, we take into account the factor 1.3 and refit the Borel window and threshold parameter for the $c c \bar{u} \bar{d}$ tetraquark state, and obtain the mass $3.91 \mathrm{GeV}$ and pole residue $2.85 \times 10^{-2} \mathrm{GeV}^{5}$, which is consistent with the values $M=3.90 \mathrm{GeV}$ and $\lambda=2.64 \times 10^{-2} \mathrm{GeV}^{5}$ in Table 1. So neglecting the perturbative $\mathcal{O}\left(\alpha_{s}\right)$ corrections cannot impair the predictive ability remarkably.

In Fig.5, we plot the masses with variations of the energy scale $\mu$ for the central values of the input parameters shown in Table 1. From the figure, we can see that the masses decrease monotonously with increase of the energy scale, it is impossible to obtain energy scale independent QCD sum rules. In this article, we choose the special values determined by the energy scale formula in a consistent way.

In Table 2, we list out the present predications compared to the values from some typical theoretical approaches, such as the simple quark model [8], heavy quark symmetry [16], lattice QCD [18, 19]. From the Table, we can see that the masses of the doubly charmed tetraquark 

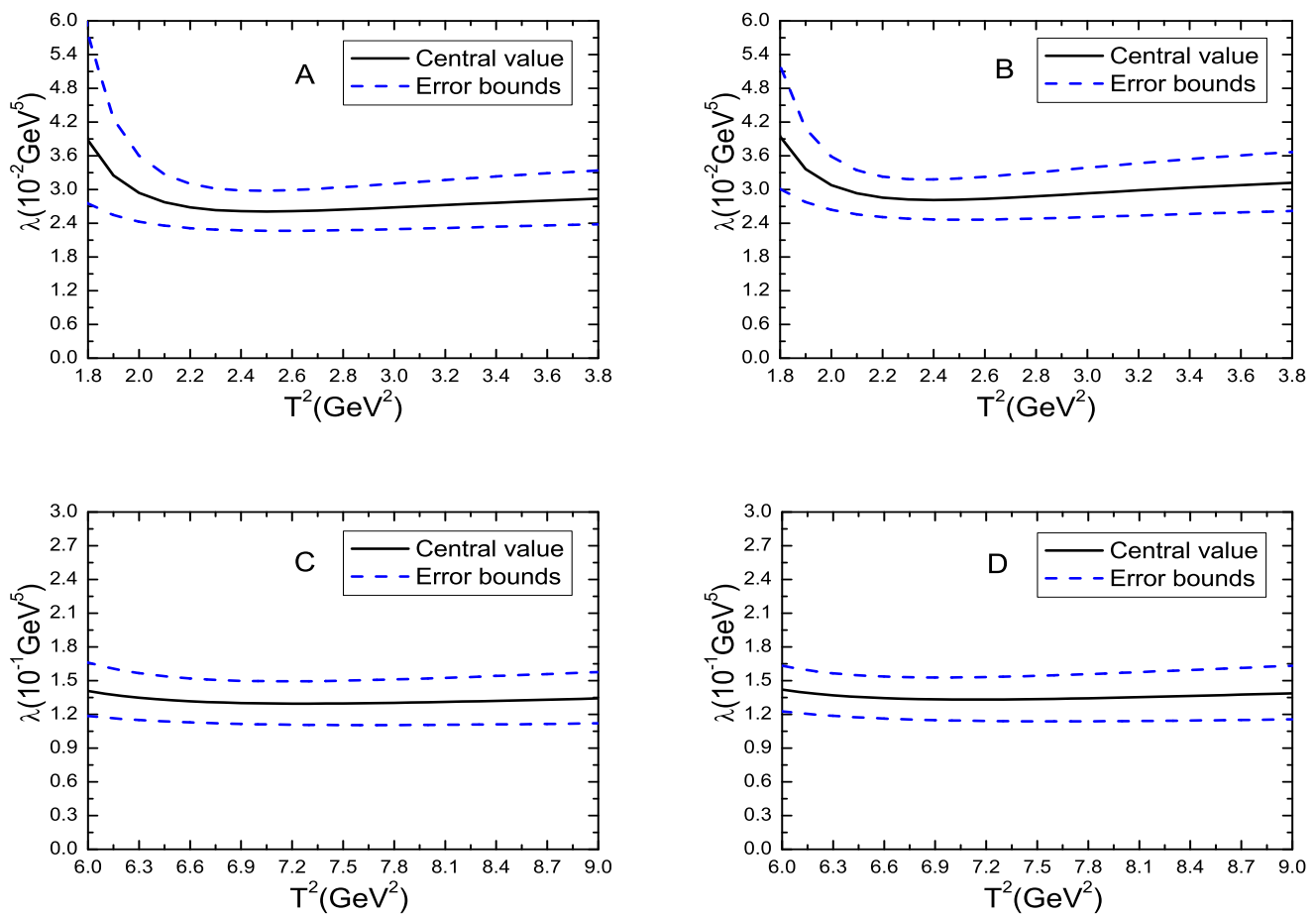

Figure 4: The pole residues with variations of the Borel parameter $T^{2}$, where the $A, B, C$ and $D$ denote the tetraquark states $c c \bar{u} \bar{d}, c c \bar{u} \bar{s}, b b \bar{u} \bar{d}$ and $b b \bar{u} \bar{s}$, respectively.
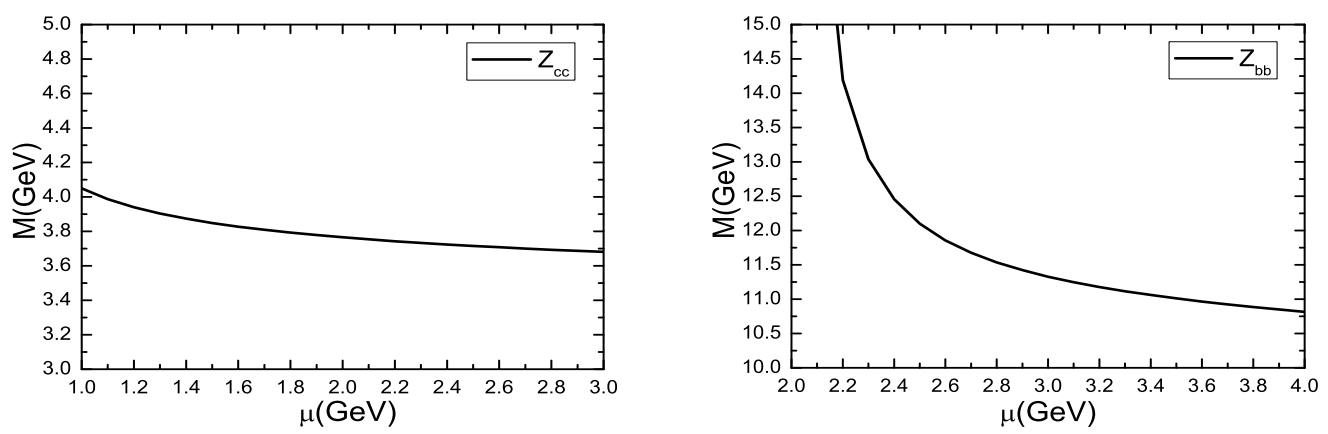

Figure 5: The masses of the $Z_{c c \bar{u} \bar{d}}$ and $Z_{b b \bar{u} \bar{d}}$ with variations of the energy scale $\mu$. 


\begin{tabular}{|c|c|c|c|c|}
\hline \hline & $c c \bar{u} d$ & $c c \bar{u} \bar{s}$ & $b b \bar{u} d$ & $b b \bar{u} \bar{s}$ \\
\hline$[\underline{8}]$ & $3.882 \pm 0.012$ & & $10.389 \pm 0.012$ & \\
\hline$[16]$ & 3.978 & 4.156 & 10.482 & 10.643 \\
\hline$[18]$ & & & $10.545_{-0.030}^{+0.038}$ & \\
\hline$[19]$ & & & $10.415 \pm 0.010$ & $10.549 \pm 0.008$ \\
\hline This work & $3.90 \pm 0.09$ & $3.95 \pm 0.08$ & $10.52 \pm 0.08$ & $10.55 \pm 0.08$ \\
\hline Thresholds & $3.875 / 3.876$ & $3.977 / 3.976$ & $10.604 / 10.604$ & $10.692 / 10.695$ \\
\hline
\end{tabular}

Table 2: The present predications compared to other theoretical works, where the Thresholds denote the two-meson thresholds $D^{0} D^{*+} / D^{+} D^{* 0}, D^{0} D_{s}^{*+} / D_{s}^{+} D^{* 0}, \bar{B}^{0} B^{*-} / B^{-} \bar{B}^{* 0}$ and $\bar{B}_{s}^{0} B^{*-} / B^{-} \bar{B}_{s}^{* 0}$, respectively, the unit is $\mathrm{GeV}$.

states lie (slightly) above the corresponding lowest meson-meson thresholds, while the masses of the doubly bottom tetraquark states lie (slightly) below the corresponding lowest meson-meson thresholds, although the predicted masses differ from each other in one way or the other.

The decays of the doubly charmed (bottom) tetraquark states $Z_{c c}\left(Z_{b b}\right)$ to the charmed-meson (bottom meson) pairs are Okubo-Zweig-Iizuka super-allowed. The two-body strong decays

$$
Z_{c c \bar{u} \bar{d}} \rightarrow D^{0} D^{*+}, D^{+} D^{* 0},
$$

are kinematically allowed, but the available phase spaces are very small, if the hadronic coupling constant $G_{Z_{c c \bar{u} \bar{d}} D D^{*}}=G_{Z_{c}(3900) D \bar{D}^{*}}=0.62 \mathrm{GeV}\left[38\right.$, then the width $\Gamma_{Z_{c c \bar{u} \bar{d}}}=0.44 \mathrm{MeV}$. Even for large hadronic coupling constant $G_{Z_{c c \bar{u} \bar{d}} D D^{*}}=10 G_{Z_{c}(3900) D \bar{D}^{*}}=6.2 \mathrm{GeV}$, the width $\Gamma_{Z_{c c \bar{u} \bar{d}}}=$ $44 \mathrm{MeV}$ is still negligible [39. The two-body strong decays

$$
Z_{c c \bar{u} \bar{s}} \rightarrow D^{0} D_{s}^{*+}, D_{s}^{+} D^{* 0},
$$

can only take place for the upper bound of the predicted mass $M_{Z_{c c \bar{u} \bar{s}}}$, the width is expected to be tiny. While the two-body strong decays

$$
\begin{aligned}
& Z_{b b \bar{u} \bar{d}} \rightarrow \bar{B}^{0} B^{*-}, B^{-} \bar{B}^{* 0}, \\
& Z_{b b \bar{u} \bar{s}} \rightarrow \bar{B}_{s}^{0} B^{*-}, B^{-} \bar{B}_{s}^{* 0},
\end{aligned}
$$

are kinematically forbidden, the $Z_{b b \bar{u} \bar{d}}$ and $Z_{b b \bar{u} \bar{s}}$ can decay weakly through $b \rightarrow c \bar{c} s$ at the quark level,

$$
\begin{aligned}
& Z_{b b \bar{u} \bar{d}} \rightarrow \bar{B}^{0} B^{*-}, B^{-} \bar{B}^{* 0} \rightarrow \gamma J / \psi K^{-} J / \psi \bar{K}^{0}, \\
& Z_{b b \bar{u} \bar{s}} \rightarrow \bar{B}_{s}^{0} B^{*-}, B^{-} \bar{B}_{s}^{* 0} \rightarrow \gamma J / \psi \phi J / \psi K^{-},
\end{aligned}
$$

the widths can be neglected safely. The doubly charmed tetraquark states may be narrow resonances; while the doubly bottom tetraquark states may be real ground tetraquark states and would establish the existence of doubly bottom tetraquarks and illuminate the role of heavy diquarks in color antitriplet as the basic constituents. According to the small or tiny widths of the lowest states, the one-pole approximation works well. We can search for the doubly heavy tetraquark states in those decays in the future.

\section{Conclusion}

In this article, we construct the axialvector-diquark-scalar-antidiquark type currents to interpolate the axialvector doubly heavy tetraquark states, and study them with QCD sum rules by carrying out the operator product expansion up to the vacuum condensates of dimension 10. In calculations, 
we take the energy scale formula as a constraint to determine the energy scales of the QCD spectral densities in a consistent way to extract the masses and pole residues. In the Borel windows, the pole dominance is satisfied and the operator product expansion is convergent, and we expect to make reliable predictions. The present predictions indicate that the two body strong decays to the charmed meson pairs are kinematically allowed, while two body strong decays to the bottom meson pairs are kinematically forbidden, we can search for the axialvector doubly charmed (bottom) tetraquark states in strong (weak) decays in the future.

\section{Acknowledgements}

This work is supported by National Natural Science Foundation, Grant Number 11775079.

\section{References}

[1] A. De Rujula, H. Georgi and S. L. Glashow, Phys. Rev. D12 (1975) 147; T. DeGrand, R. L. Jaffe, K. Johnson and J. E. Kiskis, Phys. Rev. D12 (1975) 2060.

[2] R. Aaij et al, Phys. Rev. Lett. 119 (2017) 112001.

[3] J. R. Zhang and M. Q. Huang, Phys. Rev. D78 (2008) 094007; Z. G. Wang, Eur. Phys. J. A45 (2010) 267; Z. G. Wang, Eur. Phys. J. C68 (2010) 459; S. Narison and R. Albuquerque, Phys. Lett. B694 (2011) 217; T. M. Aliev, K. Azizi and M. Savci, Nucl. Phys. A895 (2012) 59; T. M. Aliev, K. Azizi and M. Savci, J. Phys. G40 (2013) 065003; H. X. Chen, Q. Mao, W. Chen, X. Liu and S. L. Zhu, Phys. Rev. D96 (2017) 031501.

[4] J. P. Ader, J. M. Richard and P. Taxil, Phys. Rev. D25 (1982) 2370; S. Zouzou, B. SilvestreBrac, C. Gignoux and J. M. Richard, Z. Phys. C30 (1986) 457.

[5] H. J. Lipkin, Phys. Lett. B172 (1986) 242; L. Heller and J. A. Tjon, Phys. Rev. D35 (1987) 969; J. Carlson, L. Heller and J. A. Tjon, Phys. Rev. D37 (1988) 744; B. Silvestre-Brac and C. Semay, Z. Phys. C57 (1993) 273; D. M. Brink and Fl. Stancu, Phys. Rev. D57 (1998) 6778; B. A. Gelman and S. Nussinov, Phys. Lett. B551 (2003) 296; D. Janc and M. Rosina, Few Body Syst. 35 (2004) 175; N. Barnea, J. Vijande and A. Valcarce, Phys. Rev. D73 (2006) 054004; J. Vijande, E. Weissman, A. Valcarce and N. Barnea, Phys. Rev. D76 (2007) 094027.

[6] S. Pepin, F. Stancu, M. Genovese and J. M. Richard, Phys. Lett. B393 (1997) 119; D. Ebert, R. N. Faustov, V. O. Galkin and W. Lucha, Phys. Rev. D76 (2007) 114015; J. Vijande, A. Valcarce and N. Barnea, Phys. Rev. D79 (2009) 074010; Y. Yang, C. Deng, J. Ping and T. Goldman, Phys. Rev. D80 (2009) 114023; T. F. Carames, A. Valcarce and J. Vijande, Phys. Lett. B699 (2011) 291; A. Czarnecki, B. Leng and M. B. Voloshin, Phys. Lett. B778 (2018) 233.

[7] F. Buccella, H. Hogaasen, J. M. Richard and P. Sorba, Eur. Phys. J. C49 (2007) 743; S. Q. Luo, K. Chen, X. Liu, Y. R. Liu and S. L. Zhu, Eur. Phys. J. C77 (2017) 709.

[8] M. Karliner and J. L. Rosner, Phys. Rev. Lett. 119 (2017) 202001.

[9] A. Esposito, M. Papinutto, A. Pilloni, A. D. Polosa and N. Tantalo, Phys. Rev. D88 (2013) 054029 .

[10] F. S. Navarra, M. Nielsen and S. H. Lee, Phys. Lett. B649 (2007) 166.

[11] Z. G. Wang, Y. M. Xu and H. J. Wang, Commun. Theor. Phys. 55 (2011) 1049.

[12] M. L. Du, W. Chen, X. L. Chen and S. L. Zhu, Phys. Rev. D87 (2013) 014003. 
[13] A. V. Manohar and M. B. Wise, Nucl. Phys. B399 (1993) 17.

[14] M. Karliner and S. Nussinov, JHEP 1307 (2013) 153.

[15] T. Mehen, Phys. Rev. D96 (2017) 094028.

[16] E. J. Eichten and C. Quigg, Phys. Rev. Lett. 119 (2017) 202002.

[17] Z. S. Brown and K. Orginos, Phys. Rev. D86 (2012) 114506; Y. Ikeda et al, Phys. Lett. B729 (2014) 85; P. Bicudo, K. Cichy, A. Peters, B. Wagenbach and M. Wagner, Phys. Rev. D92 (2015) 014507; A. Peters, P. Bicudo, L. Leskovec, S. Meinel and M. Wagner, PoS LATTICE2016 (2016) 104.

[18] P. Bicudo, J. Scheunert and M. Wagner, Phys. Rev. D95 (2017) 034502.

[19] A. Francis, R. J. Hudspith, R. Lewis and K. Maltman, Phys. Rev. Lett. 118 (2017) 142001.

[20] M. A. Shifman, A. I. Vainshtein and V. I. Zakharov, Nucl. Phys. B147 (1979) 385; Nucl. Phys. B147 (1979) 448.

[21] L. J. Reinders, H. Rubinstein and S. Yazaki, Phys. Rept. 127 (1985) 1.

[22] S. Narison, Camb. Monogr. Part. Phys. Nucl. Phys. Cosmol. 17 (2002) 1.

[23] Z. G. Wang and T. Huang, Phys. Rev. D89 (2014) 054019.

[24] Z. G. Wang, Eur. Phys. J. C74 (2014) 2874.

[25] Z. G. Wang, Commun. Theor. Phys. 63 (2015) 466.

[26] Z. G. Wang and T. Huang, Nucl. Phys. A930 (2014) 63.

[27] Z. G. Wang and T. Huang, Eur. Phys. J. C74 (2014) 2891; Z. G. Wang, Eur. Phys. J. C74 (2014) 2963.

[28] L. Maiani, F. Piccinini, A. D. Polosa and V. Riquer, Phys. Rev. D89 (2014) 114010.

[29] L. Maiani, F. Piccinini, A. D. Polosa and V. Riquer, Phys. Rev. D71 (2005) 014028.

[30] P. Pascual and R. Tarrach, "QCD: Renormalization for the practitioner", Springer Berlin Heidelberg (1984).

[31] P. Colangelo and A. Khodjamirian, hep-ph/0010175

[32] C. Patrignani et al, Chin. Phys. C40 (2016) 100001.

[33] Z. G. Wang, Eur. Phys. J. C76 (2016) 70.

[34] Z. G. Wang, Eur. Phys. J. C76 (2016) 387; Z. G. Wang, Commun. Theor. Phys. 66 (2016) 335.

[35] Z. G. Wang, Commun. Theor. Phys. 63 (2015) 325.

[36] Z. G. Wang, Eur. Phys. J. C77 (2017) 78; Z. G. Wang, Eur. Phys. J. A53 (2017) 19.

[37] Z. G. Wang and Z. H. Yan, Eur. Phys. J. C78 (2018) 19.

[38] Z. G. Wang and J. X. Zhang, Eur. Phys. J. C78 (2018) 14.

[39] Z. G. Wang, Int. J. Mod. Phys. A30 (2015) 1550168. 